\title{
Establishment and Analysis of Face Recognition Model Based on Least Square Method
}

\author{
Fan Yang ${ }^{1,2}$ and Xuewen Tan ${ }^{1}$ \\ ${ }^{1}$ School of Mathematics and Computer Science, Yunnan Minzu University, Kunming 650500, China \\ ${ }^{2}$ Key Laboratory of IOT Application Technology of Universities in Yunnan Province, Yunnan Minzu University, Kunming \\ 650500, China
}

\begin{abstract}
Face recognition technology is studied to realize the reconstructed image after many years. The principal component analysis of local facial feature extraction using MATLAB images, two value curve, corrosion and boundary contour extraction, vertical and horizontal projection contour extraction after the picture is compared, obtained by the least square method fitting the corresponding function. The validity and accuracy of the model are verified by simulation.
\end{abstract}

Keywords-gray level processing; feature extraction; projection; least square method

\section{INTRODUCTION}

With the rapid development of informational and scientific technology, face recognition technology has become an important research direction. Such as pattern recognition, artificial intelligence, computer, image processing, the development of these technologies has played an important role on face recognition technology, it has been widely used in various fields, for example, financial, military, public security, frontier defense, aerospace, electric power, education, medical and other fields, as a result, face recognition has important research significance and huge space for development. But the photograph of a face image will be affected by some uncertain factors, such as sunlight, posture, age, place of residence and so on. These external factors also are adverse to extract facial features. Our country is developing and perfecting human face recognition technology, it should be under the premise and basis of human face feature extraction. Therefore, the steady and efficient feature extraction method is kind of realistic and significant.

In this paper, the main purpose of face recognition technology is to match feature extraction method and image to extract features in images of the same person at different ages. First of all, preprocessing two different images at different ages, namely gray. Then extracting features of images preprocessed through MATLAB knowledge after preprocessing, and feature extraction of a grayscale image mainly includes three parts, eyes, nose, and mouth.

\section{THE DESCRIPTION OF SySTEMATIC THEORY MODEL}

Literature [1] is a method to mainly study the human positive face recognition, which introduces the Eigenface method, the elastic matching (Elastic) method based on Wavelet feature, the shape and gray of deformable Model
(Flexible Model) and traditional modeling analysis methods. Literature [2] introduces a series of principles and methods of image processing. Literature [3] has studied a series of digital image processing and pattern recognition. Literature [4] introduces a traditional basic theory of active contour model, curve evolution theory, the basic principle of level set method and the main ideas of narrow-band algorithm, which are based on level set method and combined with the Snake model and facial contour extraction of narrow-band algorithm. Literature [5] introduces MATLAB usage about image processing. Literature [6] mainly studies age problems in face recognition and realizes the face recognition of Robustness for age change. Literature [7] propose a new face recognition method based on Singular Value Decomposition (SVD) and Hmbedded Hidden Markov Model (EHMM). Through the literature, we can see that the significance of face recognition, of course, there are a lot of problems, data loss and other factors will affect the accuracy of the results, it also inspire our research interest of face recognition.

This paper mainly considers the feature extraction method and image matching as the purpose for research, and studies the image feature extraction of the same person at different ages. From "feature extraction", first of all, we extract facial features of the same person at different ages and use the MATLAB code implements feature extraction on the image preprocessed, after processing features extracted, based on the MATLAB code, we achieve horizontal and vertical projection to get the corresponding data chart, and will compare fitting functions received by the Least Square Method.

\section{MATHEMATICS Modeling}

\section{A. Images Preprocessing}

The preprocessing of selected images is mainly based on the method of face detection, which mainly includes the following two steps.

1. Image grayscale: due to different backgrounds of different images, the background of the image should be processed with grayscale at first, and the brightness of all images should be adjusted appropriately. Image grayscale processing is to get the corresponding grayscale through collected RGB images. The grayscale method is relatively simple in early image grayscale processing, it usually is the maximum or mean value of the three components of color image RGB. Images grayscaled with mean value will get more 
smooth, the brightness of images of taking the maxminum is high. Formula[1] is now more commonly used:

$$
\mathrm{I}=0.3 \mathrm{P}+0.59 \Gamma+0.11 \mathrm{~B}
$$

due to the details of the original image are easy to be ignored.

2. Image binarization: a digital image is stored as a bitmap in a computer, which is a rectangular lattice.And each dot represents a pixel. In image binarization, the image pixel gray value is set from 0 to 255 (in general, white grey value is defined as 255 value and black grey value is defined as 0 value, so the light-shade level between black to white is evenly divided into 256 level), due to light-shade degree of each image depends on the specific grey value (gray level). Image binarization is very important in face recognition, when it appears contour of the target, at the same time it greatly reduces the image data and saves the computer storage space of images, as a result, it improves the computation efficiency of the image.

Formula [1] shows the brightness value of the grayscale, $\mathrm{R}$ is for color image component value of red, $G$ represents the value of color image component green, B represent blue components of color image value. By comparing the grayscale of the formula transformation can be a good reaction the brightness information of the original image[3].

Grayscale images are shown in Image 1.

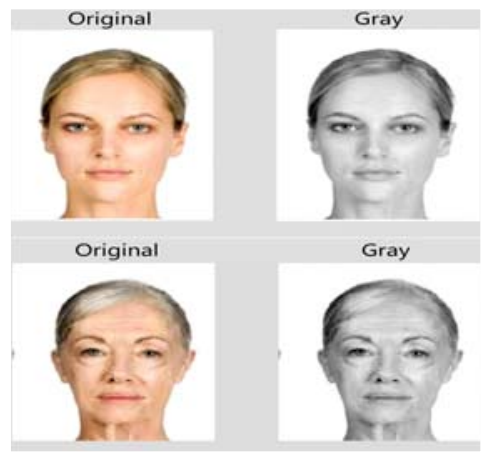

FIGURE I. IMAGES 1

\section{B. Features Extraction}

Feature extraction is mainly a process whereby analyzes and extracts image information through computer, also can be understood as another process that converts the visual information of images into the quantitative form computer can recognize and deal with. Feature extraction mainly includes the following steps: Firstly, in order to the nest operation can be easy, the image should be preprocessed to make the image tidy. Secondly, the image should be normalized. Finally, the feature vectors that best reflect the image are extracted.

On the one hand, feature extraction does not have a more precise definition, which is often defined by the problem itself or the type of application. Feature is the starting point of many computer image analysis algorithms, which makes feature extraction a primary operation in image processing. Whether this pixel can represent a feature is often determined by checking each pixel. The size or contrast of the object has great influence on the extraction of image features. In general, the image will be further studied when the size of the object is different or the contrast is large. At the same time, feature extraction is "repeatable", which is also a feature.

On the other hand, photos of the same people at different ages need to be extracted the same parts, such as when extracting eyes feature, we should extract twice: a person's feature in young and old age. Only in this way, the results are comparable.

In this paper, the algorithm of automatic extraction feature is improved, and the improved one can manually extract the feature, which can select the desired feature part.

Extracting features manually:

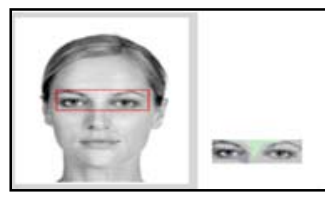

FIGURE II. IMAGES 2

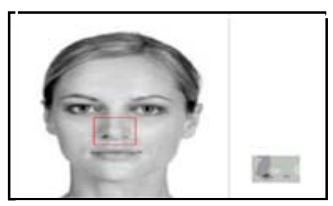

FIGURE IV. IMAGES 4

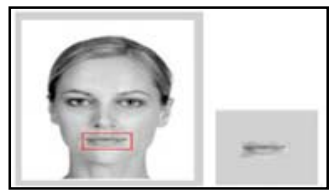

FIGURE VI. IMAGES 6

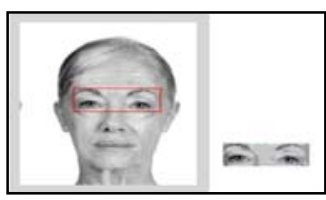

FIGURE III. IMAGES 3

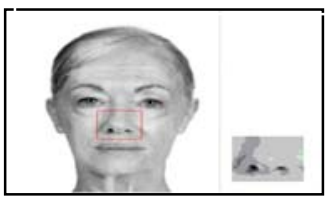

FIGURE V. IMAGES 5

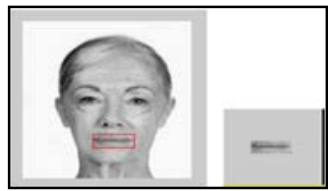

FIGURE VII. IMAGES 7

\section{Processing of Feature Region}

The edge of the image is the most obvious place of gray change, which can clearly show the basic characteristics of images. Facial contour [4] can be clearly marked and delineate the dividing line between the five sense organs. Facial contour can best reflect the basic features of face, and it can be applied to face recognition. The basic idea of facial contour extraction is to find a area where region gray of a image varies larger in the original image will be extracted, there are two basic principles: Firstly, finding the area in which the first-order derivatives of brightness is greater than a specified threshold; Secondly, finding the area where the second derivatives of brightness have zero crossings.

Then to process the feature extraction in these steps: image binarization processing, corrosion, extracting boundary contour. Image 8, 10, 12 represents repectively young people's eyes, nose and mouth. Image 9, 11, 13 respectively is on behalf of the old people's eyes, nose and mouth. As shown in the following image. 


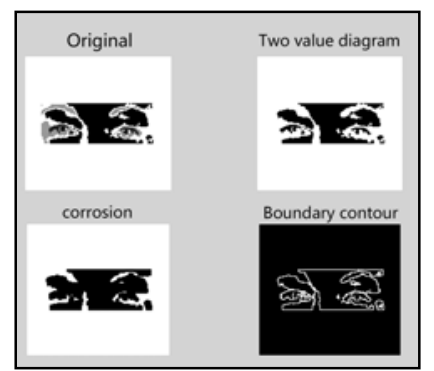

FIGURE VIII. IMAGE8

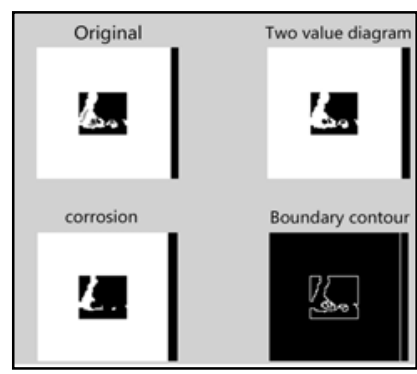

FIGURE X. IMAGE10

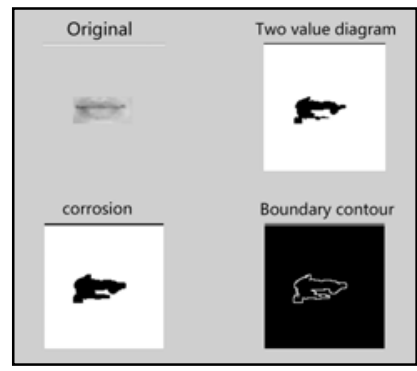

FIGURE XII. IMAGE12

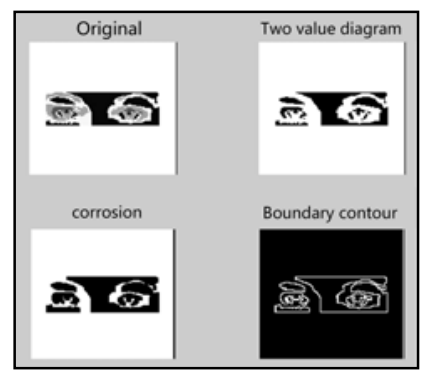

FIGURE IX. IMAGE9

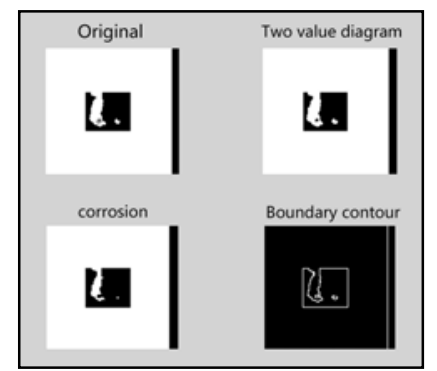

FIGURE XI. IMAGE11

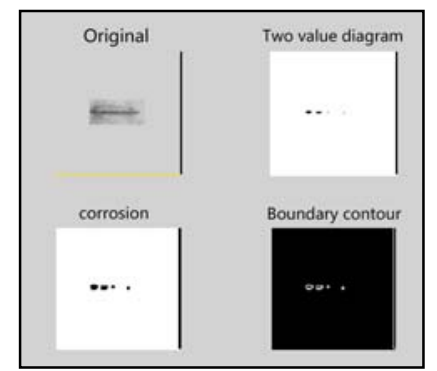

FIGURE XIII. IMAGE13

\section{Projection}

Making a projection about feature contour, it can greatly reduce the influence of the noise on the image, so that features can be more directly reflected in the form of data through the comparison between images. The image signal is converted to digital signal to facilitate comparison between the images. Image 15, table I, and table II represent the projection of people in their youth, and old age shown in image16, table III and table IV.

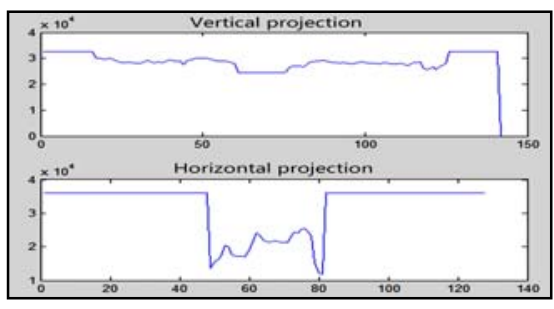

FIGURE XIV. IMAGE14

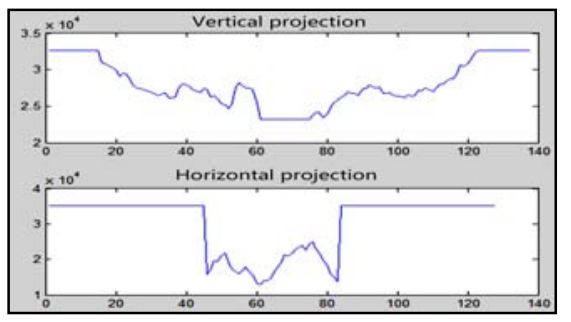

FIGURE XV. IMAGE15

\section{E. Least Square Method}

Operating the least square fitting of projection coordinate table I, II, III and IV to get the following fitting function curve:

Image 16 and 17 are the characteristic vertical and horizontal projection fitting curves for young people respectively. Image 18 and 19 are the characteristics of vertical and horizontal projection fitting curves for elderly people respectively.

Curve function of vertical projection of young people:

$$
f(x)=0.0002 x^{4}-0.0031 x^{3}+0.0653 x^{2}-0.7713 x+3.9027
$$

Curve function of vertical projection of elderly people:

$$
f(x)=0.0003 x^{4}-0.0057 x^{3}+0.0977 x^{2}-0.7811 x+5.8830
$$

Curve function of horizontal projection of young people:

$$
f(x)=-0.0001 x^{4}+0.0653 x^{3}-0.1393 x^{2}+1.2513 x-4.4814
$$

Curve function of horizontal projection of elderly people:

$$
f(x)=-0.0002 x 4+0.0445 x 3-0.0651 \times 2+0.6822 x-3.4479
$$

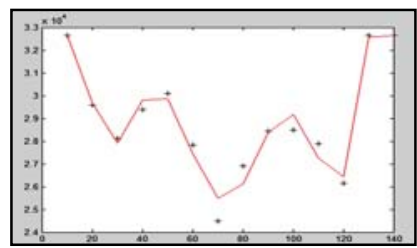

FIGURE XVI. IMAGE16

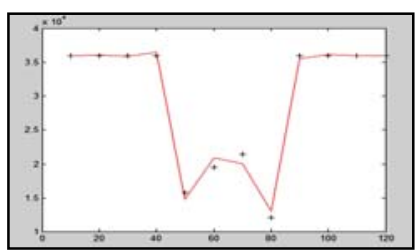

FIGURE XVIII. IMAGE18

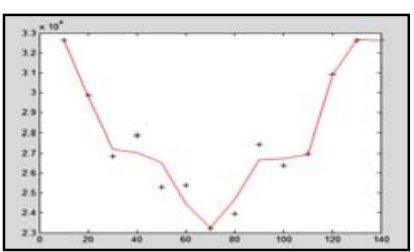

FIGURE XVII. IMAGE17

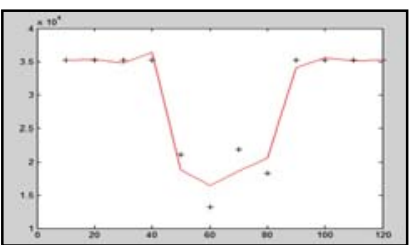

FIGURE XIX. IMAGE19

\section{EXPERIMENT}

Experiment data are shown in the following tables. 
TABLE I. VERTICAL PROJECTION COORDINATE OF EYES FEATURE IN YOUNG AGE

\begin{tabular}{|c|c|c|c|c|}
\hline $\boldsymbol{x}$ & 10 & 20 & 30 & 40 \\
\hline $\boldsymbol{y}$ & $3.596 \mathrm{E}+04$ & $3.596 \mathrm{E}+04$ & $3.596 \mathrm{E}+04$ & $3.596 \mathrm{E}+04$ \\
\hline $\boldsymbol{x}$ & 50 & 60 & 70 & 80 \\
\hline $\boldsymbol{y}$ & $1.576 \mathrm{E}+04$ & $1.949 \mathrm{E}+04$ & $2.142 \mathrm{E}+04$ & $1.204 \mathrm{E}+04$ \\
\hline $\boldsymbol{x}$ & 90 & 100 & 110 & 120 \\
\hline $\boldsymbol{y}$ & $3.596 \mathrm{E}+04$ & $3.596 \mathrm{E}+04$ & $3.596 \mathrm{E}+04$ & $3.596 \mathrm{E}+04$ \\
\hline
\end{tabular}

TABLE II. HORIZONTAL PROJECTION COORDINATE OF EYES FEATURE IN YOUNG AGE

\begin{tabular}{|c|c|c|c|c|}
\hline $\boldsymbol{x}$ & 10 & 20 & 30 & 40 \\
\hline $\boldsymbol{y}$ & $3.264 \mathrm{E}+04$ & $2.960 \mathrm{E}+04$ & $2.813 \mathrm{E}+04$ & $2.939 \mathrm{E}+04$ \\
\hline $\boldsymbol{x}$ & 50 & 60 & 70 & 80 \\
\hline $\boldsymbol{y}$ & $3.010 \mathrm{E}+04$ & $2.783 \mathrm{E}+04$ & $2.448 \mathrm{E}+04$ & $2.692 \mathrm{E}+04$ \\
\hline $\boldsymbol{x}$ & 90 & 100 & 110 & 120 \\
\hline $\boldsymbol{y}$ & $2.844 \mathrm{E}+04$ & $2.849 \mathrm{E}+04$ & $2.789 \mathrm{E}+04$ & $2.614 \mathrm{E}+04$ \\
\hline $\boldsymbol{x}$ & 130 & 140 & & \\
\hline$y$ & $3.264 \mathrm{E}+04$ & $3.624 \mathrm{E}+04$ & & \\
\hline
\end{tabular}

TABLE III. VERTICAL PROJECTION COORDINATE OF EYES FEATURE IN OLD AGE

\begin{tabular}{|c|c|c|c|c|}
\hline $\boldsymbol{x}$ & 10 & 20 & 30 & 40 \\
\hline $\boldsymbol{y}$ & $3.264 \mathrm{E}+04$ & $2.986 \mathrm{E}+04$ & $2.684 \mathrm{E}+04$ & $2.788 \mathrm{E}+04$ \\
\hline $\boldsymbol{x}$ & 50 & 60 & 70 & 80 \\
\hline $\boldsymbol{y}$ & $2.530 \mathrm{E}+04$ & $2.538 \mathrm{E}+04$ & $2.322 \mathrm{E}+04$ & $2.394 \mathrm{E}+04$ \\
\hline $\boldsymbol{x}$ & 90 & 100 & 110 & 120 \\
\hline $\boldsymbol{y}$ & $2.743 \mathrm{E}+04$ & $2.637 \mathrm{E}+04$ & $2.696 \mathrm{E}+04$ & $3.090 \mathrm{E}+04$ \\
\hline $\boldsymbol{x}$ & 130 & 140 & & \\
\hline$y$ & $3.264 \mathrm{E}+04$ & $3.264 \mathrm{E}+04$ & & \\
\hline
\end{tabular}

TABLE IV. HORIZONTAL PROJECTION COORDINATE OF EYES FEATURE IN OLD AGE

\begin{tabular}{|c|c|c|c|c|}
\hline $\boldsymbol{x}$ & 10 & 20 & 30 & 40 \\
\hline $\boldsymbol{y}$ & $3.519 \mathrm{E}+04$ & $3.519 \mathrm{E}+04$ & $3.519 \mathrm{E}+04$ & $3.519 \mathrm{E}+04$ \\
\hline $\boldsymbol{x}$ & 50 & 60 & 70 & 80 \\
\hline $\boldsymbol{y}$ & $2.111 \mathrm{E}+04$ & $1.321 \mathrm{E}+04$ & $2.185 \mathrm{E}+04$ & $1.825 \mathrm{E}+04$ \\
\hline $\boldsymbol{x}$ & 90 & 100 & 110 & 120 \\
\hline $\boldsymbol{y}$ & $3.519 \mathrm{E}+04$ & $3.519 \mathrm{E}+04$ & $3.519 \mathrm{E}+04$ & $3.519 \mathrm{E}+04$ \\
\hline
\end{tabular}

\section{CONCLUSION}

In this paper, extracting face feature by using the least square method, mainly taking eyes area as an example to establish related model, then based on the Matlab platform, through processing image data, getting the corresponding fitting function, further analyzing the function, finally judging whether this is the same person in two different images. In view of this, it can be seen that the mathematical model has a high recognition rate and it has the advantages of simple algorithm, convenient implementation, and the ability to manually extract feature range and so on. At the same time, it can be seen that the method used in this paper has a better effect on face recognition technology from the fitting function.

\section{ACKNOWLEDGMENT}

This paper was supported by State Ethnic Affairs Commission education reform project (Practical teaching reform of mathematical modeling in Universities for Nationalities under the background of big data), Yunnan Minzu University education reform project (The research of university mathematics teaching reform and the cultivation of innovative talents under the Internet plus) and Yunnan Minzu University education reform project (mathematical modeling teaching team).

\section{REFERENCES}

[1] Zhang cui-ping, Su guang-da, A review of facial recognition technology [J], Journal of Chinese Graphic Graphics (A), 2000, 5 (11): 885-894.

[2] Sun jin-guang, Zhang wen-bin, Zhu shi-an. Methods and implementations of image grayscale processing [A]. Journal of Engineering Technology of Liaoning University: Natural Science Edition, 2002.

[3] Shen ting-zhi, Wang wei-jiang, Yan xue-mei. Digital image processing and pattern recognition [M], edition 2. Beijing: Beijing Institute of Technology Press, 2007.

[4] Pang dong-hui, research on face contour extraction based on narrowband set level model, December 15, 2012.

[5] Zhao jing, Dan qi, mathematical modeling and experiment, Beijing, Higher Education press, 2014, edition 4.

[6] Wang jun-yan, face recognition of age change, July 24, 2016, edition B13.

[7] Chen jun, zhang jian-ming, face recognition at age change based on the singular value [A], September, 2008. 